\title{
Assessing the uncertainty of tree height and aboveground biomass from terrestrial laser scanner and hypsometer using airborne LiDAR data in tropical rainforests
}

\begin{abstract}
Tree height is one of the key parameters for estimating forest aboveground biomass (AGB). Traditionally, the tree height is measured by hypsometers, which are widely used to validate terrestrial laser scanner (TLS) and Airborne LiDAR (ALS). However, the measurements from hypsometers are subject to huge uncertainties in comparison with TLS and ALS. The error associated with the height measurements propagate into the AGB estimation models, and eventually downgrade the accuracy of estimated AGB and the subsequent carbon stock. In this article, we test the use of hypsometer, TLS and ALS in a tropical lowland rainforest to measure the height $(\mathrm{H})$ and diameter at breast height $(\mathrm{DBH})$ and take Airborne LiDAR as a benchmark with high accuracy and fidelity in height measurements. The results revealed that, the field height measured by hypsometer underestimated the tree height with root-mean-square error (RMSE) of 3.11, whereas the TLS underestimated height with RMSE of 1.61, when Airborne LiDAR was used as a benchmark to validate the field measurement and TLS. Due to significant differences in derived height measurements, the AGB and carbon stock also varied remarkably with values of 146.33 and $68.77 \mathrm{Mg}$ from field measurements, 170.86 and $80.31 \mathrm{Mg}$ from TLS, 179.85 and $84.53 \mathrm{Mg}$ using the Airborne LiDAR. Considering the Airborne LiDAR measurement as the most accurate, the AGB and carbon stock from field measurement represent $85.55 \%$ of total AGB and carbon stock estimation from Airborne LiDAR. Meanwhile, TLS measurements reflect $95.02 \%$ of AGB and carbon stock benchmarked with the measurements from Airborne LiDAR data. The results demonstrate the huge uncertainty in height measurement of large trees in comparison with small trees indicated by the significant differences. It was concluded that AGB and carbon stocks are sensitive to height measurement errors derived from various methods for measuring the tree height, the size of trees as large trees are difficult to measure height using hypsometer and TLS as opposed to small trees that are visible as well as the forest conditions. Compared with Airborne LiDAR, TLS achieved the higher accuracy of height estimation (R $2=0.91$ with RMSE of 1.61 ) than the hypsometer ( R $2=0.61$ with RMSE of 3.11).
\end{abstract}

Keyword: Accuracy; Airborne LiDAR; Biomass; Carbon stock; Hypsometer and error; Terrestrial laser scanner (TLS); Tree height; Tropical forest 